\title{
SUPERMATRIX IN AHP: UNDOING EIGENVECTOR NORMALIZATION
}

\author{
Stan Schenkerman \\ - University of Bridgeport, Bridgeport, CT 06601 (203) 576-4384 * \\ , ABSTRACT
}

It is shown that for concordant supermatrices the supermatrix approach succeeds by simply undoing normalizations required by the AHP. For these supermatrices the same results are more easily obtained by leaving the measurement data unnormalized and using the standard weighted-sums approach. A subsequent paper at this symposium shows that for discordant supermatrices even the supermatrix approach yields arbitrary and ambiguous results.

\section{INTRODUCTION}

In the AHP [5] rank reversal (and other distortions of overall priorities) due to the addition or deletion of an alternative is the source of much controversy. Opponents of AHP contend that rank reversals and distortions demonstrate a fundamental flaw. Some opponents even propose methods for modifying hierarchical composition to avoid such rank reversals and distortions [1] [2] [6] [9] [10]. In contrast, proponents of AHP argue that rank reversals and distortions are acceptable, or that in the cases cited the criteria are not independent of the alternatives--that the supermatrix approach, rather than hierarchical composition, must be used. These proponents maintain that hierarchical composition is a special case of the supermatrix. approach, and that the supermatrix approach is always applicable.

This paper ${ }^{1}$ shows that for concordant supermatrices (all supermatrices based upon objective absolute measurements and some based upon subjective relative measurements), the supermatrix approach proffered by AHP proponents succeeds by simply undoing the eigenvector normalizations required by AHP (now recognized as the cause of rank reversals and priority distortions). For these supermatrices the same results can be obtained more easily by leaving the measurement data unnormalized and using the standard weighted-sums approach. A subsequent paper at this symposium [8] shows that for discordant supermatrices (all remaining supermatrices) even the supermatrix approach is incorrect. Thus, the supermatrix approach is unnecessary in the one case and invalid in the other.

After defining concordance and discordance and stating Proposition 1: the denormalization effect of concordant supermatrices (which is proved in the Appendix), this paper provides illustrations using examples from an oft cited paper by Harker and Vargas (H\&V) [4]. ${ }^{2}$ Concomitantly, three tenets of AHP propounded in the H\&V paper are critiqued:

1. Rank reversal occurring from the addition or deletion of an alternative is a "fact of life".

2. Copies of alternatives should be excluded from the list of alternatives under consideration.

3. Hierarchical composition is a special case of the supermatrix approach. The supermatrix approach is always valid, but when applicable, hierarchical composition yields correct results more simply.

It is shown that the first two tenets are invalid and the third tenet requires extreme conditions. A corollary to Proposition 1 shows that the "independent" weights required for hierarchical composition generally cannot be independent of the alternatives. ${ }^{3}$ 


\section{CONCORDANT AND DISCORDANT SUPERMATRICES}

The first subsection of the Appendix reviews pertinent background of supermatrix theory, and provides the nomenclature for defining concordant and discordant supermatrices. Note that the element of matrix $\mathbf{X},\left(x_{i . j}\right)$, is the absolute measurement of Alternative $i$ on Criterion $\mathbf{j}, \mathbf{A}=$ $\left(a_{i i}\right)$ is the matrix of relative scores of the alternatives with respect to the criteria, and $\mathbf{B}=\left(b_{j i}\right)$ is the matrix of relative scores of the criteria with respect to the alternatives.

With absolute measurements in $\mathbf{X}$, the matrices $\mathbf{A}$ and $\mathbf{B}$ can be derived from $\mathbf{X}$ (either directly or from objective pairwise-comparison matrices). In the absence of absolute measurements, i.e., for relative measurements, there is no measurement matrix $\mathbf{X}$ and $\mathbf{A}$ and $\mathbf{B}$ must be developed from pairwise-comparison matrices assessed by the decision maker (DM) subjectively. In the former case it is possible to regenerate $\mathbf{X}$ (within a constant of proportionality) from $\mathbf{A}$ and $\mathbf{B}$, and the regenerated $\mathbf{X}$ allows derivation of that same $\mathbf{A}$ and that same $\mathbf{B}$. In the latter case, it is possible to compute an induced $\mathbf{X}$ from $\mathbf{A}$ and $\mathbf{B}$, but the $\mathbf{A}$ and $\mathbf{B}$ derived from the induced $\mathbf{X}$ may not be the same as those used to induce $\mathbf{X}$.

DEFINITION 1. Matrices $\mathbf{A}$ and $\mathbf{B}$, and hence the supermatrix comprising them, are concordant if and only if the matrix $\mathbf{X}$ they induce yields that same $\mathbf{A}$ and that same $\mathbf{B}$. Otherwise, they are discordant.

Concordance indicates compatibility between $\mathbf{A}$ and $\mathbf{B}$; discordance, lack of compatibility. It follows that $\mathbf{A}$ and $\mathbf{B}$ derived from an $\mathbf{X}$ determined from absolute measurements will always be concordant. Those developed from relative measurements can be discordant. Specifically, letting $C_{j}=\sum_{i} x_{i j}$ be the sum of Column $j$ and $R_{i j}=\sum_{i j} x_{i j}$ be the sum of Row $i, a_{i j}=x_{i j} / C_{j}$ and $\mathrm{b}_{\mathrm{ji}}=\mathrm{x}_{\mathrm{ij}} / \mathrm{R}_{\mathrm{i}}$ if and only if $\mathbf{A}$ and $\mathbf{B}$ are concordant (see $\left.\nmid \dot{\gamma} \mathrm{j}\right)$.

The main implication of concordance can now be stated. As shown in the Appendix,

PROPOSITION 1. The concordant-supermatrix approach works by undoing the eigenvector normalizations (normalization of the columns of $\mathrm{A}$ ) required by AHP. ${ }^{4}$

Accordingly, there is no need to bother with AHP normalization in the first place. Indeed, there is no need to bother with the supermatrix at all. Under absolute (objective) measurements the overall priorities can be more easily computed directly from $\mathrm{X}$ as the vector of normalized row sums. Under concordant relative (subjective) measurements the nonpreemptive linear goal program used to determine concordance/discordance yields the overall priorities as standard output (see [8]).

\section{UNDOING EIGENVECTOR NORMALIZATION}

The undoing of eigenvector normalization is illustrated using the Dyer and Wendell [3] example from $\mathrm{H} \& \mathrm{~V}$ [4, pp. 1396-1398]. As shown in Table la, there are three and four alternatives being evaluated under four criteria. Measurements are absolute (objective). Since the criteria are assumed equally important, the overall priorities (Columns 6 and 8, for three alternatives and four alternatives) are simply the normalized row sums (the row sums divided by 52 and 70 , respectively). Columns 7 and 9 show that whether alternative $A_{4}$ is absent or present, the rank order of the other three alternatives does not change. There is no rank reversal from the addition or deletion of $\mathrm{A}_{4}$.

Tables $1 \mathrm{~b}$ and 1c show what happens using the standard AHP paradigm, hierarchical composition. Using either pairwise comparison matrices (or since the matrices are consistent, Theorem 7.22 in [5]), eigenvectors of alternatives are obtained which have been normalized by dividing by the column sums. Then, using equal weights for the criteria, the overall priorities 
are computed. In both cases the overall priorities and rank order are different from those in Table $l a$ and rank reversal among $A_{1}-A_{3}$ occurs upon the addition or deletion of $A_{4}$.

Which priorities and rank orders are correct, Tables $1 \mathrm{~b}$ and $1 \mathrm{c}$ or Table la? Since the measurements are absolute (objective) Table 1a is correct. Suppose that (unlike in [3] and [4]) each alternative represents a different path of four links between common beginning and ending nodes, that the criteria represent the links $\left(C_{1}\right.$ the first link, $C_{2}$ the second, etc.), and that the individual measurements are the link lengths. If the focus (overall objective) is total path length, criteria weights are equal and each aiternative's total path length is given by its row sum in Table 1a. Certainly, the presence or absence of $A_{4}$ cannot affect the total path length or the rank order of the other alternatives. Yet, using AHP's hierarchical composition, there is just such an effect.

$\mathrm{H} \& \mathrm{~V}$ contend that in this case independence of criteria from alternatives is violated and therefore the supermatrix approach, not hierarchical composition, should be used [4, p. 1397]. They then show that with the supermatrix, the four-alternative case gives the correct overall priorities (Column 8 in Table 1a.) But, other than their assertion that the supermatrix approach, not hierarchical composition, should be used, H\&V do not explain why the supermatrix approach is successful. As illustrated below, the reason is given in Proposition 1 .

\section{The Concordant-Supermatrix Approach.}

Figure 1 shows the (concordant) supermatrix, $\mathbf{W}$, for the three-alternative case (which $\mathrm{H} \& \mathrm{~V}$ omit): Raising $W$ to a sufficiently high odd power (here 25 is used) yields supermatrix $\mathbf{W}^{25}$. Each column of the southwest partition gives the correct overall priorities--those in Column 6 of Table 1a. Thus the concordant-supermatrix approach (henceforth supermatrix approach) gives the correct rank order of the alternatives (Column 7).

Additionally, consider the four-alternative case, Figure 2, which H\&V do present. Again $\mathbf{W}$ is concordant and again each column in the southwest partition of $W^{25}$ gives the correct overall priorities (Column 8 in Table 1a) yielding the correct rank order (Column 9).

By giving the correct overall priorities, the supermatrix approach avoids the rank reversal and other distortions of overall priorities that are so troublesome in hierarchical composition. Accordingly, contrary to Tenet 1 , rank reversal is not a "fact of life".

How does the supermatrix approach achieve what hierarchical composition fails to achieve? According to supermatrix theory, in both Figures 1 and 2, each column in the northeast partition of $\mathbf{W}^{25}$ gives the set of independent criteria weights-weights which if premultiplied by the A matrix (the southwest partition of $W$ ) would yield the correct overall priorities directly. As illustrated in Figures 1 and 2, the $\mathbf{b}$ vector is any column of the northeast partition of $\mathbf{W}^{25}$, and the $\mathbf{b}$-vector's components are also the normalized column sums from Table 1a. That is, the numerator of component $b_{j}$ is the column sum, $C_{j}$, used to normalize the $j^{\text {th }}$ eigenvector and obtain Tables $1 \mathrm{~b}$ and $1 \mathrm{c}$. As shown in Figures 1 and 2 , these independent criteria weights undo the eigenvector normalizations: $\mathbf{A} * \mathbf{b}=\mathbf{O P R T Y}$, the vector of overall priorities. Thus, as stated earlier in Proposition 1 (and proved in the Appendix): the supermatrix approach works by undoing AHP-required eigenvector normalization.

\section{ARE COPIES LEGITIMATE?}

In another example, H\&V criticize Belton and Gear's example of rank reversal [1] by asserting that alternatives which are copies of other alternatives must be omitted from the choice set [4, pp. 1399-1400]. We now show that while hierarchical composition might fail with copies, the supermatrix approach deals with them successfully. 
The Belton and Gear example is shown in Table 2a. After comparing three alternatives on three equally weighted criteria, they add a fourth alternative, $A_{4}$, which is a copy of $A_{2}$.

Tables $2 \mathrm{~b}$ and $2 \mathrm{c}$ show what happens with AHP's hierarchical composition: the presence or absence of $A_{4}$ changes the rank order between $A_{1}$ and $A_{2}$. Curiously, unlike the previous example, in this case $H \& V$ do not defend the rank reversal by asserting violation of criteria independence. Instead, they challenge the presence of $\mathrm{A}_{4}$ because it is a copy of another alternative, claiming that copies violate one of the axioms of AHP and should be ruled out "because [a copy] does not add anything to the choice set" [4, p. 1399]. The supermatrix approach, which they fail to examine, shows that copies need not be ruled out.

Figures 3 and 4 show the supermatrix approach in the absence and presence of copy $A_{4}$. As before, the independent criteria weights (the components of the b vector, equivalently the components of each column in the northeast partition of $W^{25}$ ) undo the AHP-required eigenvector normalizations: $\mathbf{A} * \mathbf{b}=\mathbf{O P R T Y}$, the vector of overall priorities. Thus, the presence or absence of the copy does not affect the rank order of the other alternatives. In other words, contrary to Tenet 2 , using the supermatrix approach, copies are legitimate. The flaw is not in using copies, but in the basic paradigm of AHP, hierarchical composition. The flaw is the failure of hierarchical composition to do what the supermatrix approach does--undo eigenvector normalization. Indeed, the flaw is normalizing the eigenvectors in the first place.

\section{HOW SPECIAL A CASE IS HIERARCHICAL COMPOSITION?}

Although it is generally held that hierarchical composition is a special case of the supermatrix approach, it is generally not appreciated how special that special case must be.

In [4, pp. 1400-1401] H\&V try to demonstrate that hierarchical composition is equivalent to the supermatrix approach when the northeast partition of $\mathrm{W}$ consists of identical columns. As they illustrate (and as can be shown in general), the northeast partition of $W^{2 k+1}$ then also consists of the same identical columns, i.e., raising $W$ to the $2 k+1$ power does not change the northeast partition. Therefore, $H \& V$ conclude, the independent criteria weights are given by any of those identical columns.

That conclusion is misguided. For hierarchical composition to be a valid substitute for the supermatrix approach, more than those identical columns is needed. H\&V have only illustrated an artifact of raising $\mathbf{W}$ to a power: any set of identical columns (that each sum to one) will exhibit the same phenomenon--the columns in the northeast partition will not change. But that alone does not make them the correct independent criteria weights. Nor can the independent criteria weights be imposed exogenously. Rather, for each alternative (for each column of the northeast partition) the criteria weights must be the components of the eigenvectors resulting from pairwise comparison matrices among criteria. It must turn out that each of these eigenvectors is the same and that each undoes the normalizations of the eigenvectors of alternatives. We now show what is required and how special the circumstances are. ${ }^{5}$

Using the nomenclature given in the first subsection of the Appendix, for identical columns, $b_{i i}$ $=b_{j 1}$ for all columns $i$ of $B=\left(b_{i j}\right)$. Since the column vector $\left(b_{i j}\right)$. must undo the normalizations of the columns of $\mathbf{X}$ and must sum to one, it is necessary that

$$
b_{j 1}=C_{j} / C, \quad j=1, \ldots n .
$$

But by definition the elements of the concordant matrix $\mathbf{B}$ are

$$
b_{j i}=x_{i j} / R_{i} \text {. }
$$


Substituting (1) into (2), the elements of $\mathbf{X}=\left(\mathrm{x}_{\mathrm{ij}}\right)$ must satisfy

$$
x_{i j}=R_{i} C_{j} / C \text {, }
$$

and the elements of the concordant matrix $A=\left(a_{i j}\right)$ must satisfy

$$
a_{i j}=x_{i j} / C_{j}=R_{i} / C=R_{i} / R \text {. }
$$

Thus, the entire matrix $\mathbf{X}=\left(x_{i j}\right)$ must satisfy (arbitrarily in terms of $\left.x_{1,1}\right)$.

$$
\left[\begin{array}{r}
x_{1,1} \\
\left(R_{2} / R_{1}\right) x_{1,1} \\
\left(R_{3} / R_{1}\right) x_{1,1} \\
\vdots \\
\vdots
\end{array}\right.
$$

$$
\begin{array}{r}
\left(C_{2} / C_{1}\right) x_{1,1} \\
\left(R_{2} / R_{1}\right)\left(c_{2} / C_{1}\right) x_{1,1} \\
\left(R_{3} / R_{1}\right)\left(C_{2} / C_{1}\right) x_{1,1}
\end{array}
$$$$
\left(c_{3} / c_{1}\right) x_{1}
$$$$
\left(R_{2} / R_{1}\right)\left(C_{3} / C_{1}\right) x_{1,1}
$$$$
\left(R_{3} / R_{1}\right)\left(C_{3} / C_{1}\right) x_{1,1}
$$

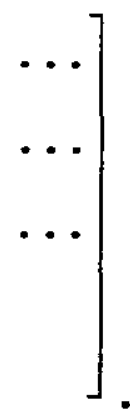

Therefore,

1. The columns of $\mathbf{B}$ must be identical.

2. Each element of the columns of $\mathbf{B}$ must cancel the corresponding column sum of $\mathbf{X}$.

3. The columns of $\mathbf{A}$ must be identical.

More fundamentally, items 1-3 imply:

4. The rows of $\mathbf{X}$ must be proportional to each other. The constant of proportionality must be the ratio of their row sums.

5. The columns of $\mathbf{X}$ must be proportional to each other. The constant of proportionality must be the ratio of their column sums.

Merely imposing identical columns of $\mathbf{B}$ without satisfying all of the foregoing conditions violates supermatrix theory. Even when each of the identical columns of $\mathbf{B}$ undoes the column normalizations of $\mathbf{X}$ (so the resulting global priorities are correct), if the other conditions are not satisfied, it is not AHP. It is some ad hoc procedure, which could have been avoided by not normalizing in the first place. Thus, contrary to Tenet 3 , hierarchical composition is not just a special case of the supermatrix approach--it is a very special case.

That hierarchical composition is generally inappropriate is even more evident from the following:!

COROLLARY TO PROPOSITION 1. Criteria weights cannot be imposed exogenously, since they must undo the AHP normalizations of the columns of A. Thus, criteria weights are usually dependent upon the alternatives. Therefore, independence is rare; hierarchical composition is seldom applicable. 


\section{FINAL REMARKS}

AHP cannot be a credible decision-making aid with subjective relative measurements without first establishing its validity with objective absolute measurements (for which answers are known independently). We have shown that for concordant $\mathbf{A}$ and $\mathbf{B}$ matrices, and in contrast to widely accepted tenets of AHP--

1. Rank reversal is not such a "fact of life".

2. Copies of alternatives are legitimate.

3. Hierarchical composition is applicable only under highly special conditions.

4. AHP's supermatrix approach is not necessary. As it succeeds by undoing the AHPrequired normalizations of the eigenvectors of alternatives, the same results can be obtained more easily by leaving the measurement data unnormalized and using the standard weighted-sums approach. ${ }^{6}$

A subsequent paper at this symposium [8] shows that for discordant $\mathbf{A}$ and $\mathbf{B}$ matrices the supermatrix approach yields arbitrary and ambiguous priorities. Therefore, the supermatrix approach is unnecessary for concordant matrices and incorrect for discordant matrices. This means that AHP itself is unnecessary under concordance and invalid under discordance.

\section{ENDNOTES}

${ }^{1}$ An early and abridged version of which appeared as [7].

${ }^{2}$ In that paper H\&V responded to Belton and Gear [1] [2] and to Dyer and Wendell [3], early critics of AHP because of rank reversal due to the addition or deletion of alternatives.

${ }^{3}$ The term independent is not clearly defined in the AHP literature. Rather, it is defined operationally as characterizing weights that allow the use of hierarchical composition. Although the term is controversial, space does not permit further discussion of the issue here.

${ }^{4}$ Harker and Vargas mathematically state a related proposition [4, pp. 1398-1399], but offer no proof and apparently overlook the significance that eigenvector normalization is being undone.

${ }^{5}$ The following assumes that the criteria are equally important to the focus. For criteria that are not equally important see the last subsection of the Appendix.

${ }^{6}$ We have only illustrated and proved this for two-level hierarchies, but if AHP is defective with two levels it is at least suspect with more levels.

\section{APPENDIX: PROOF OF PROPOSITION 1}

\section{Nomenclature and the Supermatrix}

Let $\mathrm{X}=\left(\mathrm{x}_{\mathrm{ij}}\right)$ be an nxm matrix where $\mathrm{x}_{\mathrm{ij}}$ is the absolute measurement of Alternative $\mathrm{i}$ on Criterion $j$. Let $C_{j}=\sum_{i} x_{i j}$ be the sum of Column $j, R_{i}=\Sigma_{j} x_{i j}$ the sum of Row $i$, and $C=$ $\Sigma_{j} C_{j}=R=\Sigma_{i} R_{i}=\sum_{i j} x_{i j}$ be column, row and overall totals.

Let $\mathbf{A}=\left(\mathrm{a}_{\mathrm{ij}}\right)$ be the nxm matrix whose elements are $\mathrm{a}_{\mathrm{ij}}=\mathrm{x}_{\mathrm{ij}} / \mathrm{C}_{\mathrm{i}}$, i.e., are column normalized. Let $\mathbf{B}=\left(b_{\mathrm{j}}\right)$ be the mxn matrix whose elements are $b_{\mathrm{jij}}=\mathrm{x}_{\mathrm{ij}} / \mathrm{R}_{\mathrm{i}}$, i.e., are also column normalized. Since each of their columns sums to one, $\mathbf{A}$ and $\mathbf{B}$ are column stochastic. 
As $\mathbf{A}$ is the matrix of relative scores of the alternatives on the criteria and $\mathbf{B}$ the matrix of relative scores of the criteria on the alternatives (assuming equally important criteria--an assumption dropped later), the square supermatrix, $\mathbf{W}$, is given by

$$
\boldsymbol{W}=\left[\begin{array}{c|c}
\mathbf{0} & \mathbf{B} \\
-\mathrm{A} & -- \\
\mathbf{0}
\end{array}\right] .
$$

Multiplying $W$ by itself an odd number of times yields

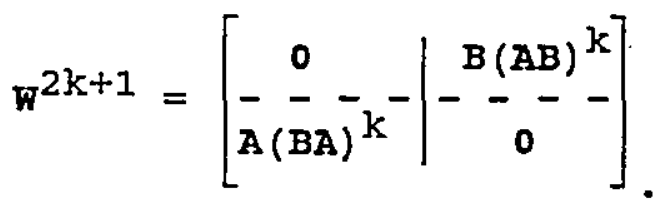

For $k$ sufficiently large the columns of $\mathbf{B}(\mathbf{A B})^{k}$ become identical as do the columns of $\mathbf{A}(\mathbf{B A})^{k}$. From supermatrix theory, each column of $\mathbf{B}(\mathbf{A B})^{\mathbf{k}}$ is the vector of independent criteria weights which, if premultiplied by $\mathbf{A}$ would yield the overall priorities without resorting to the supermatrix, i.e., by hierarchical composition directly. Each column of $\mathbf{A}(\mathbf{B A})^{\mathrm{k}}$ is the vector of overall priorities. Note each column of $(\mathbf{B A})^{\mathbf{k}}$ equals each column of $\mathbf{B}(\mathbf{A B})^{\mathbf{k}}$,. i.e., is the vector of independent criteria weights.

\section{Determination of $\mathbf{B}(\mathbf{A B})^{k}$}

If B's columns are identical each element $b_{j j}=b_{j h}$, all $i$ and $h$. Therefore, $x_{i j} / R_{i}=x_{h i j} / R_{h}$. Cross multiplying and summing on $h$ yields ${ }^{3} \mathrm{Rx}_{\mathrm{ij}} \stackrel{{ }^{2}}{=} \mathrm{R}_{i} \mathrm{C}_{\mathrm{i}}$. Using $\mathrm{C}=\mathrm{R}$, each element of $\mathrm{b}$, and hence $\mathbf{B}(\mathbf{A B})^{k}$, is given by $b_{j i}=x_{i j} / R_{i}=C_{j} / C$, which was to be proved (for $B$ with identical columns).

Now assume that $\mathbf{B}$ 's columns are not identical. Then for $\mathbf{B}(\mathbf{A B})^{\mathbf{k}}$ to have identical columns requires that $(\mathbf{A B})^{k}$ have identical columns. Let $G^{k} \equiv(A B)^{k}$. Then for $G^{k}$ to have identical columns each column of $\mathbf{G}^{\mathbf{k}}$ must be the eigenvector of $\mathbf{G}, \mathbf{Y}$, associated with the eigenvalue one. This follows from the fact that $\mathbf{G}$ and $\mathbf{G}^{\mathbf{k}}$ are column stochastic (since $\mathbf{A}$ and $\mathbf{B}$ are). Thus we need $\mathbf{Y}$ such that $\mathbf{G Y}=\mathbf{Y}$ and $\mathbf{G}^{\mathbf{k}}=(\mathbf{Y} \mathbf{Y} \mathbf{Y} \ldots)$. In particular, we need to show that $Y={ }_{i}\left(y_{i}\right)=\left(R_{i} / R\right)$, where $R_{i}$ and $R$ were defined above, is that eigenvector.

Letting $\mathbf{G}:=\left(\mathrm{g}_{\mathrm{ij}}\right)$, we have from $\mathbf{G Y}=\mathbf{Y}$

$$
\Sigma_{\mathrm{j}} \mathrm{g}_{\mathrm{ij}} \mathrm{y}_{\mathrm{j}}=\mathrm{y}_{\mathrm{i}}, \quad \mathrm{i}=1, \ldots, \mathrm{n},
$$

which, as is well known, does not yield a unique solution. If, however, we also impose

$$
\Sigma_{\mathrm{i}} \mathrm{y}_{\mathrm{i}}=1,
$$

the solution to (A1) and (A2) is unique. Now,

and (A1) becomes

$$
\begin{aligned}
& g_{i j}=\Sigma_{h} a_{i h} b_{h j}=\Sigma_{h}\left(x_{i h} / C_{h}\right)\left(x_{j h} / R_{j}\right) \\
& \sum_{j} \Sigma_{h}\left(x_{i h} / C_{h}\right)\left(x_{j h} / R_{j}\right) y_{j}=y_{i}, \quad i=1, \ldots, n .
\end{aligned}
$$


Let us try $y_{j}=R_{j} / R$. Then (A3) becomes

$$
\sum_{\mathrm{j}} \Sigma_{\mathrm{h}}\left(\mathrm{x}_{\mathrm{ih}} / \mathrm{C}_{\mathrm{h}}\right)\left(\mathrm{x}_{\mathrm{jh}} / \mathrm{R}_{\mathrm{j}}\right)\left(\mathrm{R}_{\mathrm{j}} / \mathrm{R}\right)=\mathrm{R}_{\mathrm{i}} / \mathrm{R}, \quad \mathrm{i}=1, \ldots, \mathrm{n} .
$$

But the left side of (A4) is

$$
\sum_{\mathrm{j}} \Sigma_{\mathrm{h}}\left(\mathrm{x}_{\mathrm{ih}} / \mathrm{C}_{\mathrm{h}}\right)\left(\mathrm{x}_{\mathrm{jh}} / \mathrm{R}\right)=\Sigma_{\mathrm{h}}\left(\mathrm{x}_{\mathrm{ih}} / \mathrm{C}_{\mathrm{h}}\right)\left(\mathrm{C}_{\mathrm{h}} / \mathrm{R}\right)=\Sigma_{\mathrm{h}}\left(\mathrm{x}_{\mathrm{ih}} / \mathrm{R}\right)=\mathrm{R}_{\mathrm{i}} / \mathrm{R}
$$

Thus (A4) is satisfied, which satisfies (A1). Furthermore, $y_{i}=R_{i} / R$ satisfies (A2). Therefore $y_{i}=R_{i} / R$ is the unique solution of (A1) and (A2).

According to supermatrix theory, the vector of independent criteria weights, $\mathbf{w}$, is any column of $\mathbf{B}(\mathbf{A B})^{k}$. But we have just shown $(\mathbf{A B})^{k}$ to be $(\mathbf{Y} Y \mathbf{Y} \ldots)$. Thus, $\mathbf{w}=\left(w_{j}\right)=\mathbf{B Y}$ where

$$
w_{j}=\Sigma_{i} b_{j j} y_{i}=\Sigma_{i}\left(x_{i j} / R_{i}\right)\left(R_{i} / R\right)=\Sigma_{i} x_{i j} / R=C_{j} / R=C_{j} / C \text {. }
$$

Now, Row $i$ of $A w$ is the overall priority, $p_{i}$, of Alternative $i$ and is given by

$$
\Sigma_{\mathrm{j}} \mathrm{a}_{\mathrm{ij}} \mathrm{C}_{\mathrm{j}} / \mathrm{C}=\Sigma_{\mathrm{j}}\left(\mathrm{x}_{\mathrm{ij}} / \mathrm{C}_{\mathrm{j}}\right)\left(\mathrm{C}_{\mathrm{j}} / \mathrm{R}\right)=\Sigma_{\mathrm{j}} \mathrm{x}_{\mathrm{ij}} / \mathrm{R}=\mathrm{R}_{\mathrm{i}} / \mathrm{R} \text {, }
$$

from which it is clear that, as was to be shown, the criteria weights, (A5), generated by the supermatrix approach undo the normalizations of the columns of $\mathbf{X}$.

\section{Determination of (BA)}

By a similar argument it can be shown directly that the $j^{\text {th }}$ row of the identical columns of $(B A)^{k}$ is given by (A5), which undoes the normalizations of the columns of $\mathbf{X}$.

\section{Unequal Importance of Criteria}

The foregoing assumed that the criteria are equally important to the focus (overall objective) and, therefore, that the criteria weights for $\mathbf{B}$ could be obtained directly from $\mathbf{X}$. Suppose, however; that the criteria are not equally important. B would be developed in the same way, but some columns of $\mathbf{X}$ would first have to be rescaled to reflect the relative importance of the criteria. For instance, suppose Criterion 1 was three times as important as Criterion 2 and that Criterion 2 was twice as important as Criterion 3. Then one way of rescaling $\mathbf{X}$ would be to multiply all $x_{i 1}$ by 3 and all $x_{i 3}$ by $1 / 2$. Equivalently, $X$ could be rescaled by multiplying all $x_{i 2}$ by 2 and all $x_{i 1}$ by 6 .

\section{REFERENCES}

[1] Belton, V., \& Gear, A. E., "On a shortcoming of Saaty's method of analytic hierarchies", Omega 11/3 (1983) 228-230.

[2] Belton, V., \& Gear, A. E., "The legitimacy of rank-reversal", Omega 12/5 (1984) 513-516.

[3] Dyer, J. S. \& Wendell, R. E., "A critique of the analytic hierarchy process". Working Paper 84/85-4-24. Austin: University of Texas, Department of Management, 1985.

[4] Harker, P. T., \& Vargas, L. G., "The theory of ratio scale estimation: Saaty's analytic hierarchy process," Management Science 33/11 (1987) 1383-1403. 
[5] Saaty, T. L., The analytic hierarchy process. New York: McGraw-Hill, 1980. Republished by Pittsburgh, PA: University of Pittsburgh, 1988.

[6] Schenkerman, S., "Avoiding rank reversal in AHP decision-support models" European Journal of Operational Research, forthcoming.

[7] Schenkerman, S., "The supermatrix in AHP decision-support models: quintessential or unessential?" An abridged version appears in the Proceedings of the 1993 Annual Meeting of the Northeast Decision Sciences Institute, April 1993.

[8] Schenkerman, S., "The supermatrix in AHP: concordance and discordance". Third International Symposium on the Analytic Hierarchy Process, Washington D.C. (July 11-13, 1994).

[9] Schoner, B., \& Wedley, W. C., "Ambiguous criteria weights in AHP: consequences and solutions", Decision Sciences 20/3 (1989) 462-475.

[10] Schoner, B., Wedley, W. C., \& Choo, E. U., "A unified approach to AHP with linking pins". European Journal of Operational Research 64/1 (1993) 384-392. 
Table 18.

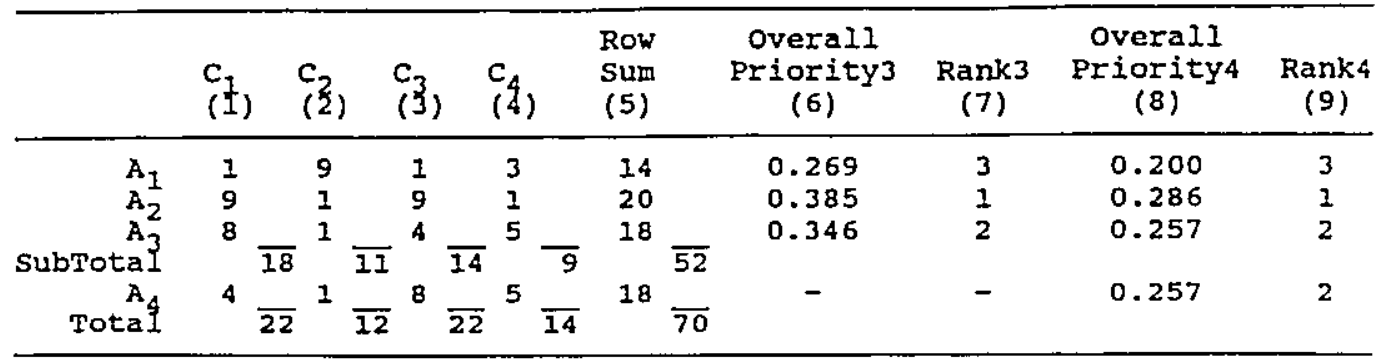

Table 2b.

\begin{tabular}{ccccccc}
\hline & $c_{1}$ & $c_{2}$ & $c_{3}$ & $c_{4}$ & $\begin{array}{c}\text { Overall } \\
\text { Priority3 }\end{array}$ & Rank3 \\
\hline $\mathrm{A}_{1}$ & $1 / 18$ & $9 / 11$ & $1 / 14$ & $3 / 9$ & 0.320 & 3 \\
$\mathrm{~A}_{2}$ & $9 / 18$ & $1 / 11$ & $9 / 14$ & $1 / 9$ & 0.336 & 2 \\
$\mathrm{~A}_{3}$ & $8 / 18$ & $1 / 11$ & $4 / 14$ & $5 / 9$ & 0.344 & 1 \\
\hline
\end{tabular}

rable Ic.

\begin{tabular}{ccccccc}
\hline & $c_{1}$ & $c_{2}$ & $c_{3}$ & $c_{4}$ & $\begin{array}{c}\text { Overal1 } \\
\text { Priority4 }\end{array}$ & Rank4 \\
\hline$A_{1}$ & $1 / 22$ & $9 / 12$ & $1 / 22$ & $3 / 14$ & 0.264 & 1 \\
$A_{2}$ & $9 / 22$ & $1 / 12$ & $9 / 22$ & $1 / 14$ & 0.243 & 3 \\
$A_{3}$ & $8 / 22$ & $1 / 12$ & $4 / 22$ & $5 / 14$ & 0.246 & 2 \\
$A_{4}$ & $4 / 22$ & $1 / 12$ & $8 / 22$ & $5 / 14$ & 0.246 & 2 \\
\hline
\end{tabular}

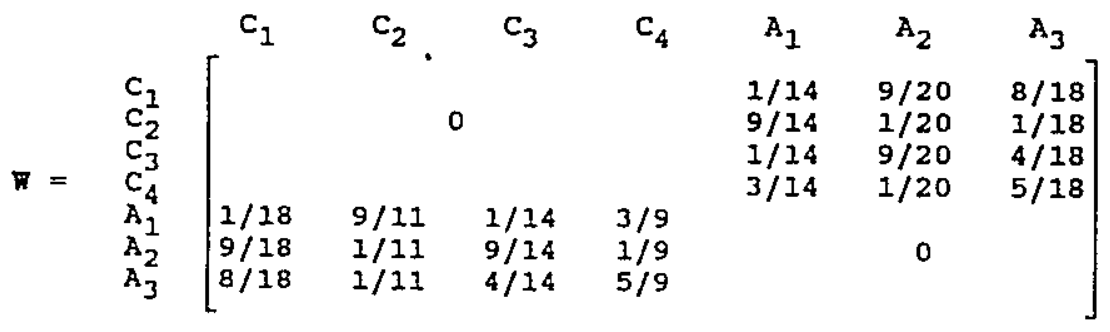

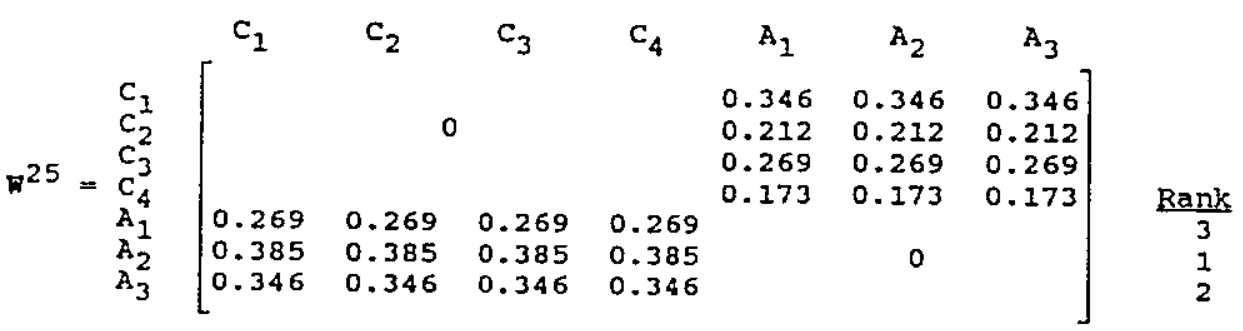

$b=\left[\begin{array}{l}0.346 \\ 0.212 \\ 0.269 \\ 0.173\end{array}\right]=\left[\begin{array}{r}18 / 52 \\ 11 / 52 \\ 14 / 52 \\ 9 / 52\end{array}\right] \quad\left[\begin{array}{llll}1 / 18 & 9 / 11 & 1 / 14 & 3 / 9 \\ 9 / 18 & 1 / 11 & 9 / 14 & 1 / 9 \\ 8 / 18 & 1 / 12 & 4 / 14 & 5 / 9\end{array}\right] *\left[\begin{array}{r}18 / 52 \\ 11 / 52 \\ 14 / 52 \\ 9 / 52\end{array}\right]=\left[\begin{array}{l}0.269 \\ 0.385 \\ 0.346\end{array}\right]$

Pigura 1 


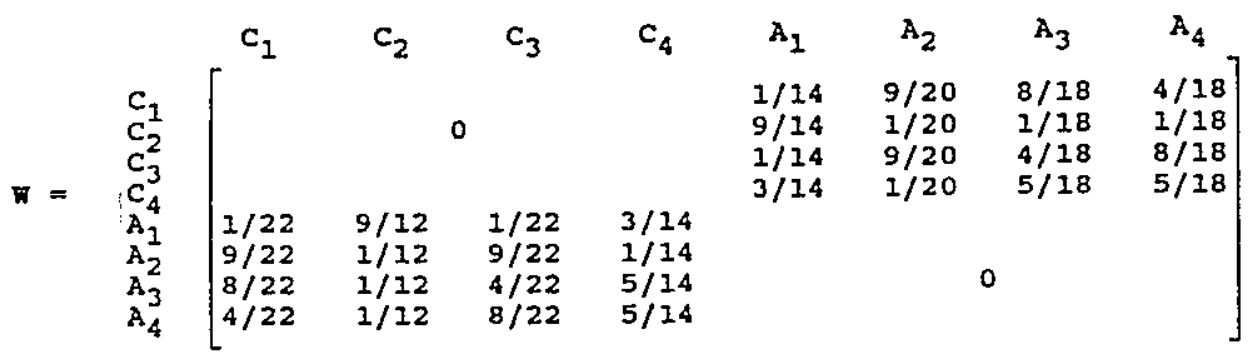

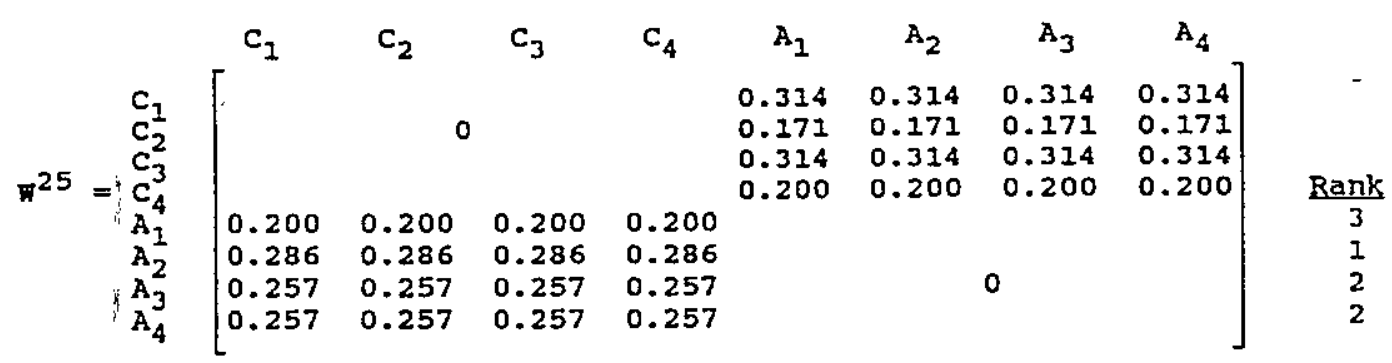

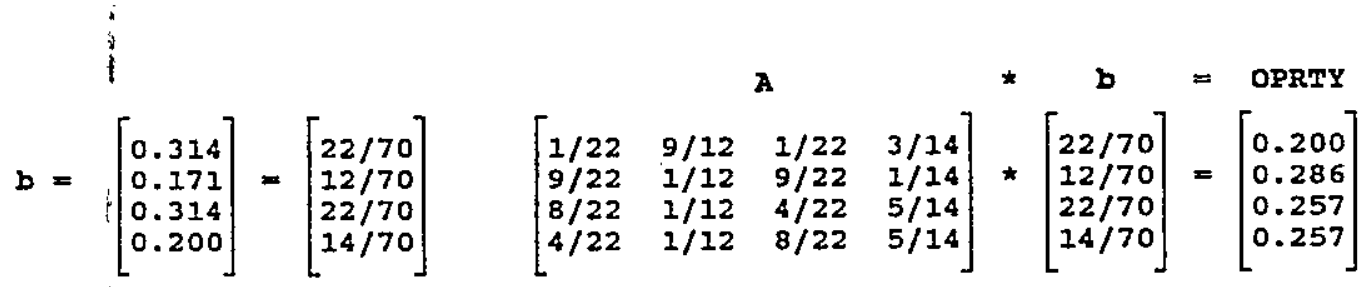

Pigure 2

Table 2a.

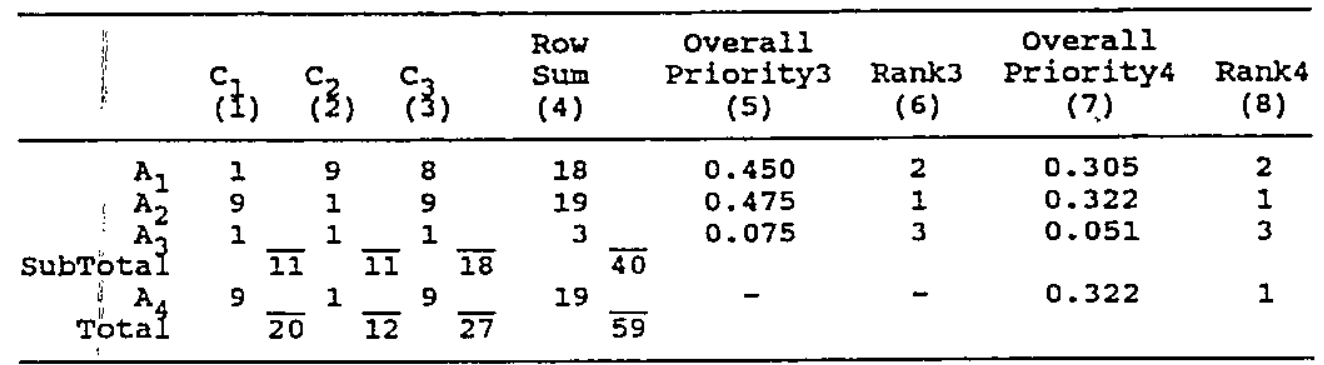

Table $2 b$.

\begin{tabular}{cccccc}
\hline & $c_{1}$ & $c_{2}$ & $c_{3}$ & $\begin{array}{c}\text { Overall } \\
\text { Priority3 }\end{array}$ & Rank3 \\
\hline$A_{1}$ & $1 / 11$ & $9 / 11$ & $8 / 18$ & 0.451 & 2 \\
$A_{2}$ & $9 / 11$ & $1 / 11$ & $9 / 18$ & 0.470 & 1 \\
$A_{3}$ & $1 / 11$ & $1 / 11$ & $1 / 18$ & 0.079 & 3 \\
\hline
\end{tabular}

Tabie $2 \mathrm{c}$.

\begin{tabular}{|c|c|c|c|c|c|}
\hline & $c_{1}$ & $c_{2}$ & $c_{3}$ & $\begin{array}{c}\text { Overall } \\
\text { Priority4 }\end{array}$ & Rank4 \\
\hline $\begin{array}{l}A_{1} \\
A_{2} \\
A_{3} \\
A_{4}\end{array}$ & $\begin{array}{l}1 / 20 \\
9 / 20 \\
1 / 20 \\
9 / 20\end{array}$ & $\begin{array}{l}9 / 12 \\
1 / 12 \\
1 / 12 \\
1 / 12\end{array}$ & $\begin{array}{l}8 / 27 \\
9 / 27 \\
1 / 27 \\
9 / 27\end{array}$ & $\begin{array}{l}0.365 \\
0.289 \\
0.057 \\
0.289\end{array}$ & $\begin{array}{l}1 \\
2 \\
3 \\
2\end{array}$ \\
\hline
\end{tabular}




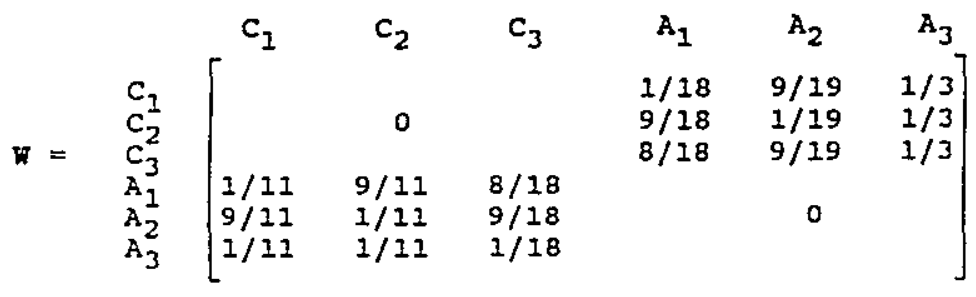

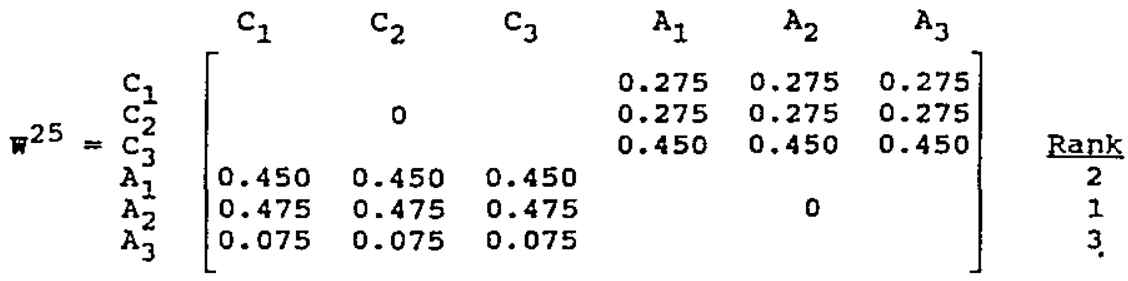

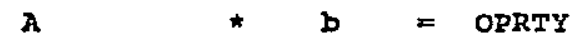

$$
b=\left[\begin{array}{l}
0.275 \\
0.275 \\
0.450
\end{array}\right]=\left[\begin{array}{l}
11 / 40 \\
11 / 40 \\
18 / 40
\end{array}\right]\left[\begin{array}{lll}
1 / 11 & 9 / 11 & 8 / 18 \\
9 / 11 & 1 / 11 & 9 / 18 \\
1 / 11 & 1 / 11 & 1 / 18
\end{array}\right] *\left[\begin{array}{l}
11 / 40 \\
11 / 40 \\
18 / 40
\end{array}\right]=\left[\begin{array}{l}
0.450 \\
0.475 \\
0.075
\end{array}\right]
$$

Figure 3

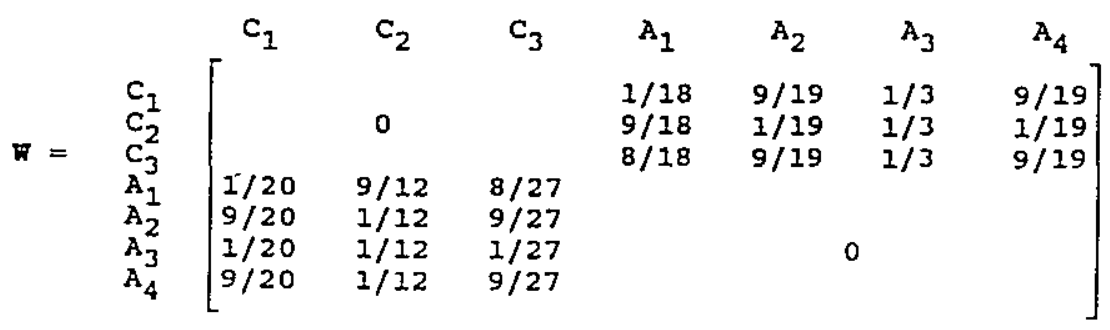

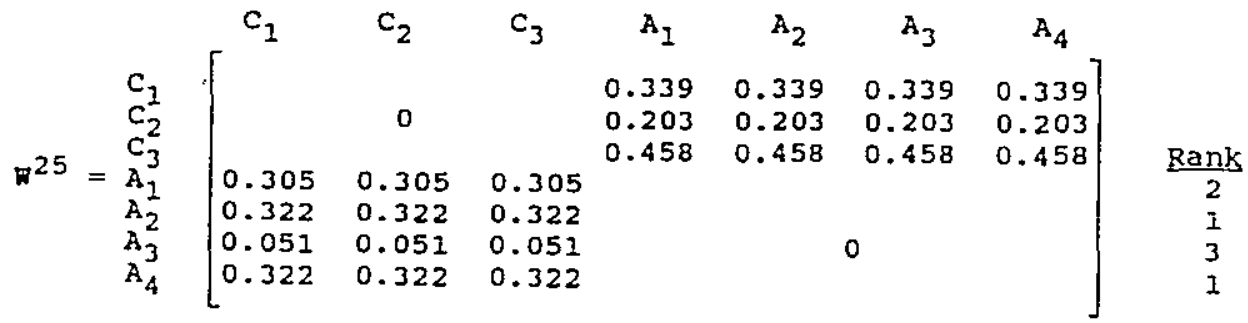

$$
\begin{gathered}
b=\left[\begin{array}{l}
0.339 \\
0.203 \\
0.458
\end{array}\right]=\left[\begin{array}{l}
20 / 59 \\
12 / 59 \\
27 / 59
\end{array}\right]=\left[\begin{array}{ccc}
1 / 20 & 9 / 12 & 8 / 27 \\
9 / 20 & 1 / 12 & 9 / 27 \\
1 / 20 & 1 / 12 & 1 / 27 \\
9 / 20 & 1.12 & 9 / 27
\end{array}\right] *\left[\begin{array}{l}
20 / 59 \\
20 / 59 \\
27 / 59
\end{array}\right]=\left[\begin{array}{l}
0.305 \\
0.322 \\
0.051 \\
0.322
\end{array}\right] \\
\text { Figure }
\end{gathered}
$$


4. Multiplicative AHP 